\section{RICYDE. Revista Internacional de Ciencias del Deporte doi: $10.5232 /$ ricyde \\ Rev. Int. cienc. deporte}

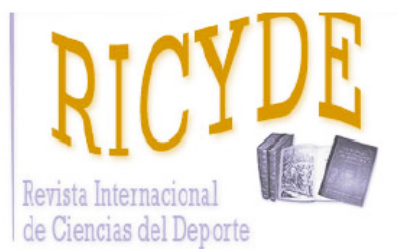

RICYDE. Revista Internacional de Ciencias del Deporte Volumen XV - Año XV

Paginas: 171-186 - ISSN: 1885-3137

Número 56 - Abril 2019

https://doi.org/10.5232/ricyde2019.05604

\title{
Efecto de un programa de ejercicio de corta duración sobre la condición física y la calidad de vida en mujeres supervivientes de cáncer de mama del ámbito rural: Estudio Piloto \\ Effect of a short duration exercise program on physical fitness and quality of life in rural breast cancer survivors: A pilot study
}

\author{
Pedro Antonio Santos-Olmo, José Fernando Jiménez-Díaz, Natalia Rioja-Collado \\ Facultad de Ciencias del Deporte de la Universidad de Castilla-La Mancha. España
}

\begin{abstract}
Resumen
El objetivo del siguiente estudio fue analizar los efectos sobre la condición física y calidad de vida (CV), de un programa de ejercicio supervisado y no supervisado en mujeres Supervivientes de Cáncer de Mama (SCM) del ámbito rural. Para ello se realizó un estudio cuasi-experimental con 114 SCM de la provincia de Ciudad Real. Mientras que el grupo control siguió su actividad física habitual, el experimental participaba en tres sesiones de ejercicio semanales; dos supervisadas y una no supervisada, donde se trabajaba movilidad articular, Capacidad Aeróbica (CA) y Resistencia Muscular (RM) de grandes grupos musculares; la duración del programa fue de cinco semanas. Las variables analizadas fueron la CA con el test UKK de dos kilómetros, RM con test funcionales de brazos y test de sentarse-levantarse de la silla. La Composición Corporal (CC) se analizó a través de impedancia bioeléctrica. La variable de CV fue medida con el cuestionario EORTC QLQ-C30 (versión 3.0). Finalizaron la intervención 21 SCM (edad $51,62 \pm 8,44$ ), ocho en el grupo experimental y 13 en el grupo control. Para las variables de la CA y RM hubo mejoras del $26,17 \%$ y $18,91 \%$, respectivamente. No se encontraron mejoras para CV, ni para la CC. Conclusiones. El programa de ejercicio supervisado y no supervisado de cinco semanas de duración ha sido insuficiente para mejorar la condición física y CV de SCM del ámbito rural.
\end{abstract}

Palabras Clave: Superviviente de Cáncer de Mama; Calidad de Vida; Capacidad Aeróbica; Resistencia Muscular; Composición Corporal.

\begin{abstract}
This study aimed to analyze the effect that a supervised and unsupervised exercise program has on the physical function and quality of life (QoL) in rural breast cancer survivors (BCS). For this, a quasi-experimental study was carried out with 114 BCS in the province of Ciudad Real. While the control group continued with its usual physical activity, the experimental group participated in three exercise sessions; two supervised and one of them unsupervised, in which they trained articular mobility, cardiorespiratory fitness (CF), and muscular resistance (MR) in large muscle groups; the duration of the program was five weeks. The variables analyzed were CF with the UKK two kilometers test, MR with the functional arms test and chair stand test. The body composition (BC) was analyzed with the bioelectrical impedance. QoL was measured using the EORTC QLQ-C30 (version 3.0) questionnaire. 21 BCS finished the intervention (aged 51,62 $\pm 8,44$ ), eight in the experimental group, and 13 in the control group. We reported improvements in CF and MR of $26,17 \%$ and $18,91 \%$ respectively. Improvements in QoL and BC were not found. Conclusions. A five weeks supervised and unsupervised exercise program was insufficient to improve physical fitness and QoL in rural BCS.
\end{abstract}

Key words: Breast Cancer Survivor; Quality of Life; Cardiorespiratory Fitness; Muscular Resistance; Body Composition.

Correspondencia/correspondence: Pedro Antonio Santos-Olmo Facultad de Ciencias del Deporte de la Universidad de Castilla-La Mancha. España Email: Pedro.SantosOlmo@uclm.es 
Santos-Olmo, P. A.; Jiménez-Díaz, J. F., y Rioja-Collado, N. (2019). Efecto de un programa de ejercicio de corta duración sobre la condición física y la calidad de vida en mujeres supervivientes de cáncer de mama del ámbito rural: Estudio Piloto. RICYDE. Revista internacional de ciencias del deporte. 56(15), 171-186.

https://doi.org/10.5232/ricyde2019.05604

\section{Introducción}

$\mathrm{E}$ l cáncer de mama es el cáncer más común en las mujeres y el segundo más común en el mundo (Bydoun, Marcato, y Dellaire, 2013). A pesar del diagnóstico y seguimiento precoz, a menudo, la superviviente de cáncer de mama (SCM) sufre las secuelas y trastornos de la enfermedad que van más allá de la fase de tratamiento (Dulko y col., 2013) con afectación multiorgánica como la condición física (alteraciones sobre la resistencia muscular o aeróbicas), fisiológicas (densidad mineral ósea) (Simonavice, Pei-Yang, Ilich, Jeong-Su, y Panton, 2011), psicológicas o de comportamiento, que limitan la capacidad para realizar tareas sociales o de la vida diaria (Campbell y col., 2012); debido en parte, a la aparición de la fatiga que puede llegar a ser muy persistente (Minton, 2013). En los países occidentalizados, el número de mujeres jóvenes con cáncer de mama (entre 15 y 39 años) tiende a aumentar, por lo que se requiere de una atención adicional al tratarse de mujeres en edad fértil con necesidad de volver al trabajo, estudios o cuidado de los hijos (Gewefel y Salhia, 2014). Para las SCM, su calidad de vida (CV) queda afectada de forma importante sin alcanzar niveles normalizados hasta los 10 años después de haber sido diagnosticada la enfermedad, aunque los peores indicadores se sitúan en el primer año (Schoormans, Czene, Hall, y Brandberg, 2015)

Existe una fuerte consistencia en cuanto a los beneficios de la práctica de ejercicio después del tratamiento (Ligibell, 2012), incrementando la resistencia muscular (RM) de los miembros superiores e inferiores y la capacidad cardiorrespiratoria (Battaglini y col., 2014). Aumenta la masa magra, modificando la composición corporal (CC) (Fernández-Lao y col., 2013).

A pesar de que el ejercicio físico se muestra como una herramienta segura sin grandes efectos adversos (Battaglini y col., 2014) y que no contribuye a la aparición o empeoramiento del linfedema (Benton, Schlairet, y Gibson, 2014), las SCM de ámbito rural realizan poca o nula AF de ocio, debido a las características del entorno que pueden ser deficitarias y de muy alto coste, el apoyo social por parte de la familia o la comunidad (Olson y col., 2014), los médicos de atención primaria y oncólogos, o las asociaciones de lucha contra el cáncer, serán un componente sencillo pero muy útil para reforzar el reclutamiento a programas de actividad física (Befort, Bennett, Christifano, Klemp, y Krebill, 2014).

Aunque existe cada vez un mayor consenso sobre el efecto beneficioso del ejercicio antes, durante y después del tratamiento del cáncer (Stout, Baima, Swisher, Winters-Stone, y Welsh, 2017) y la publicación de guías para implementar los programas de ejercicio oncológico (Schmitz y col., 2010; Segal y col., 2017), faltan evidencias sobre la recomendaciones específicas para esta patología (Chamorro y Pérez, 2012), resultando muy complejo prescribir la dosis exacta de actividad física por la variedad en la tipología, hetereogeneidad de la muestra, etapa, tratamiento del cáncer y sus consecuencias (Ruiz-Casado y col., 2014). No obstante, la prescripción de ejercicio debe tener un carácter multivariante (Musanti, 2012) incluyendo el trabajo aeróbico, que posee un potente efecto antiinflamatorio y mejora de los niveles de fatiga tanto del cáncer como de la terapia adyuvante (Cramp y Byron-Daniel, 2012), como de resistencia, por su efecto anticatabólico, que puede disminuir la caquexia asociada al tumor (Lira, Neto, y Seelaender, 2014). Así mismo, desarrolla la movilidad de los miembros superiores (Mirandola y col., 2014), pudiendo ser realizado por mujeres con linfedema, o con riesgo de padecerlo (Schmitz et al., 2010) y mejorando su autoestima e imagen corporal (Speck et al., 2010).

El objetivo general del estudio fue analizar el efecto de un programa de ejercicio supervisado y no supervisado de corta duración en un grupo de mujeres supervivientes de cáncer de mama del ámbito rural. 
Santos-Olmo, P. A.; Jiménez-Díaz, J. F., y Rioja-Collado, N. (2019). Efecto de un programa de ejercicio de corta duración sobre la condición física y la calidad de vida en mujeres supervivientes de cáncer de mama del ámbito rural: Estudio Piloto. RICYDE. Revista internacional de ciencias del deporte. 56(15), 171-186.

https://doi.org/10.5232/ricyde2019.05604

Los objetivos específicos fueron los siguientes:

1. Analizar el efecto de un programa de ejercicio supervisado y no supervisado de corta duración sobre la condición física en un grupo de mujeres supervivientes de cáncer de mama del ámbito rural.

2. Analizar el efecto de un programa de ejercicio supervisado y no supervisado de corta duración sobre composición corporal en un grupo de mujeres supervivientes de cáncer de mama del ámbito rural.

3. Analizar el efecto de un programa de ejercicio supervisado y no supervisado de corta duración la calidad de vida en un grupo de mujeres supervivientes de cáncer de mama del ámbito rural.

\section{Método}

\section{Diseño del estudio}

Se ha optado por un estudio cuasi experimental. El estudio fue aprobado por el Comité Ético de Investigación Clínica del Complejo Hospitalario "Mancha Centro" con número de acta 03/15. Las participantes adjuntaban el consentimiento firmado. El protocolo se ha elaborado teniendo en cuenta las pautas acordadas en la "Declaración de Helsinki de la Asociación Médica Mundial sobre principios éticos para las investigaciones biomédicas en seres humanos" (Fortaleza, Brasil, 2013) y siguiendo las pautas de CONSORT statement (Consolidated Standards of Reporting Trials).

\section{Participantes}

A través de los centros médicos y asociaciones contra el cáncer (AECC y Rosae de Valdepeñas) se reclutaron un total de 114 SCM de la provincia de Ciudad Real, en las localidades de Alcázar de San Juan, Argamasilla de Alba, Manzanares, Membrilla, La Solana, Santa Cruz de Mudela, Tomelloso, Valdepeñas y Villanueva de los Infantes.

La muestra debía haber padecido un cáncer de mama en sus estadios I-IIIA, haber finalizado los tratamientos de quimioterapia, radioterapia o cirugía con al menos 12 semanas de antelación (Schmitz y col., 2010), no exceder de tres años de la finalización del último tratamiento o cirugía, tener una edad entre 18 y 70 años y cumplir los criterios de inclusión. Por las características de la muestra y el entorno en el que se realizó el programa, el grupo control y el grupo experimental se conformaron atendiendo a criterios de disponibilidad y desplazamiento a las instalaciones deportivas. Entre los criterios de exclusión se encontraba enfermedad coronaria grave, patología o disfunción intelectual, casos de extrema fatiga, anemia, ataxia o enfermedad metastásica.

\section{Variables e instrumentos de medida}

Las evaluaciones se realizaron al inicio y final de programa de ejercicio (PRE y POST entrenamiento).

Capacidad Aeróbica (CA). Prueba UKK (Urho Kaleva Kekkonen Institute for Health Promotion Research) walk test o caminata de dos kilómetros (Oja y col., 2013). La prueba se realizó supervisada y con temperatura de $25^{\circ}$ según protocolo. Se utilizaron tres tapices rodantes BH Hi Power LK 6000 de motor de cuatro cv y pulsómetros Polar FT5, que posibilitaba un feedback constante del estado de las participantes.

Obteníamos en esta prueba la frecuencia cardíaca al finalizar la prueba, y tras un minuto de recuperación, Índice fitness (Oja y col., 2013), y de forma indirecta, el $\mathrm{VO}_{2 \max }$. El Índice de fitness representa las categorías de fitness o estado físico para hombres y mujeres en comparación con personas del mismo sexo de esa edad. Estas categorías se obtienen en 
Santos-Olmo, P. A.; Jiménez-Díaz, J. F., y Rioja-Collado, N. (2019). Efecto de un programa de ejercicio de corta duración sobre la condición física y la calidad de vida en mujeres supervivientes de cáncer de mama del ámbito rural: Estudio Piloto. RICYDE. Revista internacional de ciencias del deporte. 56(15), 171-186.

https://doi.org/10.5232/ricyde2019.05604

función de los valores individuales de cada participante del tiempo de realización de la prueba UKK, frecuencia cardíaca, edad e Índice de Masa Corporal (IMC), y se relacionan con los valores de referencia, donde a modo de ejemplo la media se sitúa entre los $90-110$ puntos.

Tabla 1. Categoría de Fitness según IF.

Índice Fítness

\begin{tabular}{l|l}
\hline$<70$ & Considerablemente debajo de la media \\
$70-89$ & Algo por debajo de la media \\
$90-110$ & Media \\
$11-130$ & Algo por encima de la media \\
$>130$ & Considerablemente encima de la media \\
& Nota. Fuente: Tomado de (Oja y col., 2013)
\end{tabular}

Resistencia Muscular. La RM se midió a través de test funcionales de brazos y el test de sentarse y levantarse de la silla (Pedrero-Chamizo y col., 2012; Rikli y Jones, 2013). Para la evaluación de los miembros superiores se utilizó una mancuerna de 2 '5 kg, y para los miembros inferiores, un step con soportes para que cada participante, en posición sentada, tuviera un ángulo de rodilla y cadera de $90^{\circ}$.

Composición Corporal. Analizada a través de Impedancia Bioeléctrica con el analizador TANITA TBF-300 (Tanita Corporation, Japón) “leg to leg”. Método seguro y preciso en el que una corriente de baja intensidad (150-900 ohms) pasa de pie a pie, obteniendo el porcentaje de grasa, peso e IMC.

Calidad de Vida. Medida a través del cuestionario EORTC QLQ-C30 (Aaronson y col., 1993). Se trata de un instrumento válido y fiable, usado en más de 3000 estudios para evaluar las intervenciones en pacientes con cáncer (Royo, 2011). Se compone de 30 ítems, en los que se evalúa la escala global de salud física, la capacidad funcional a través de cinco escalas (aspecto físico, autonomía, emocional, cognitiva y social), tres escalas de síntomas (fatiga, náuseas y vómitos, y dolor) además de seis ítems simples que evalúa la disnea, insomnio, pérdida de apetito, estreñimiento, diarrea y la dificultad financiera. Posee una fiabilidad adecuada con un Alpha de Crombrach entre 0,52 y 0,89 en las diferentes escalas (Royo, 2011).

\section{Intervención}

La intervención para el grupo experimental consistió en realizar durante cinco semanas, tres sesiones de ejercicio físico a la semana, dos supervisadas de al menos 75 minutos y una sesión íntegramente aeróbica y realizada de forma autónoma por las participantes. Previamente se les familiarizó durante una semana con la Escala de Borg y aprendieron a interpretar la frecuencia cardiaca que deberían alcanzar.

La sesión de actividad aeróbica libre consistió en una caminata de 60 minutos. Las participantes debían seguir las mismas pautas que las sesiones supervisadas en cuanto al calentamiento basado en movilizaciones y ligeros estiramientos, la intensidad de la actividad moderada (rango de 10 - 14 de la Escala de Borg), y finalizar con los estiramientos como en el resto de las sesiones.

Las sesiones supervisadas fueron grupales y controladas por un Licenciado en Ciencias de la Actividad Física y Deporte. Se componían de un breve calentamiento con movilizaciones y ligeros estiramientos; trabajo de RM compuesto por ocho ejercicios de grandes grupos musculares ( 2 x 10 repeticiones) (Chamorro y Pérez, 2012; Herrero y Pérez, 2011; Schmitz y col., 2010), con máquinas para los ejercicios de extensión de rodilla, extensión de rodilla y cadera, tríceps, abductor y musculatura dorsal, barras para la media sentadilla y deltoides, y 
Santos-Olmo, P. A.; Jiménez-Díaz, J. F., y Rioja-Collado, N. (2019). Efecto de un programa de ejercicio de corta duración sobre la condición física y la calidad de vida en mujeres supervivientes de cáncer de mama del ámbito rural: Estudio Piloto. RICYDE. Revista internacional de ciencias del deporte. 56(15), 171-186.

https://doi.org/10.5232/ricyde2019.05604

para el ejercicio de bíceps se utilizó barra, mancuernas y gomas; desarrollo de CA con 40 minutos de caminata; se finalizaba con el trabajo de flexibilidad. La carga era registrada, y la intensidad siempre era moderada, en el rango de 10 - 14 de la Escala de Borg.

La carga sólo fue incrementada un 5 - $10 \%$ cuando se podía realizar toda la secuencia de una sesión con soltura (Benton y col., 2014), sin provocar molestia durante la sesión, ni en el día posterior al entrenamiento. Los ejercicios se idearon con especial atención a la zona del hombro y del brazo y la amplitud del movimiento se limitaba en caso de molestia.

Al grupo control no se le dio ninguna indicación salvo mantener su nivel de actividad y cuidado habitual.

\section{Análisis Estadístico}

Se utilizó el programa estadístico SPSS v20 (paquete estadístico para las ciencias sociales). Los datos se expresaron como media y desviación estándar (media $\pm \mathrm{DE}$ ). La distribución y normalidad de las variables se analizaron mediante la prueba de Kolmogoroff Smirnoff. La prueba $\mathrm{T}$ Student para muestras independientes se utilizó para comparar los parámetros antropométricos de la muestra, y para evaluar la homogeneidad de los grupos en las variables iniciales relacionadas con $\mathrm{CA}, \mathrm{RM}, \mathrm{CC}$ y $\mathrm{CV}$ de ambos grupos (grupo control y grupo experimental). En este estudio se utilizó la prueba de medidas repetidas (ANOVA) con dos factores (tiempo y grupo) para evaluar los datos paramétricos obtenidos. En cada variable, se incluye el nivel de significación correspondiente al grupo (entre grupos), el tiempo (intragrupo) y su interacción (tiempo $\mathrm{x}$ grupo). Con el fin de evaluar el efecto de la intervención y minimizar el error de tipo I, solo se consideraron las interacciones de tiempo $\mathrm{x}$ grupo, y el nivel de significación para $\mathrm{p}<0,05$

\section{Resultados}

Para participar en el programa, 35 SCM reunían los criterios de inclusión, concluyendo el programa 21 participantes (figura 1). Todas habían sido operadas y el $60 \%$ de la muestra continuaba con tratamiento hormonal. 
Santos-Olmo, P. A.; Jiménez-Díaz, J. F., y Rioja-Collado, N. (2019). Efecto de un programa de ejercicio de corta duración sobre la condición física y la calidad de vida en mujeres supervivientes de cáncer de mama del ámbito rural: Estudio Piloto. RICYDE. Revista internacional de ciencias del deporte. 56(15), 171-186. https://doi.org/10.5232/ricyde2019.05604



Figura 1. Diagrama de flujo de la muestra.

Las participantes de los dos grupos no presentaron diferencias significativas en las variables de edad, peso IMC y porcentaje de grasa, siendo homogéneos y comparables (Tabla 2).

Tabla 2. Parámetros antropométricos de la muestra.

\begin{tabular}{lccc}
\hline Variables & Grupo & Pre & $\boldsymbol{p}$ \\
\hline Edad & Control & $52,62 \pm 8,33$ & 0,60 \\
\cline { 2 - 4 } & Experimental & $50,63 \pm 8,56$ & \\
\hline Peso (kg) & Control & $68,49 \pm 7,32$ & 0,55 \\
\cline { 2 - 4 } & Experimental & $60,56 \pm 10,52$ & 0,40 \\
\hline IMC & Control & $26,63 \pm 3,39$ & \\
\cline { 2 - 4 } & Experimental & $25,20 \pm 4,18$ & 0,34 \\
\hline \% Grasa & Control & $34,45 \pm 5,13$ & \\
\cline { 2 - 4 } & Experimental & $31,81 \pm 7,42$ &
\end{tabular}

* $\mathrm{p}<0,05$; IMC: Índice de Masa Corporal; \% Grasa: Porcentaje de grasa corporal.

\section{Capacidad Aeróbica}

A pesar de que ha habido variables que se han comportado positivamente, como es el caso del pulso al finalizar la prueba UKK, o la recuperación tras un minuto tras la prueba UKK, no se han constatado mejoras significativas en ninguna de las variables de la CA (Tabla 3). 
Santos-Olmo, P. A.; Jiménez-Díaz, J. F., y Rioja-Collado, N. (2019). Efecto de un programa de ejercicio de corta duración sobre la condición física y la calidad de vida en mujeres supervivientes de cáncer de mama del ámbito rural: Estudio Piloto. RICYDE. Revista internacional de ciencias del deporte. 56(15), 171-186.

https://doi.org/10.5232/ricyde2019.05604

Tabla 3. Efecto de un programa de ejercicio supervisado y no supervisado de cinco semanas de duración sobre las pruebas de Capacidad Aeróbica.

\begin{tabular}{|c|c|c|c|c|c|c|}
\hline Variables & Grupo & Pre & Post & $\begin{array}{c}P \text { para efecto } \\
\text { Grupo }\end{array}$ & $\begin{array}{l}P \text { para efecto } \\
\text { Tiempo }\end{array}$ & $\begin{array}{c}\text { P para efecto } \\
\text { interacción Grupo } \\
\text { x Tiempo }\end{array}$ \\
\hline \multirow[t]{2}{*}{$\mathrm{VO}_{2} \max$} & Control & $13,23 \pm 15,60$ & $15,11 \pm 11,31$ & 0,40 & $0,02 *$ & 0,24 \\
\hline & Experimental & $15,89 \pm 12,10$ & $21,52 \pm 7,29$ & & & \\
\hline \multirow[t]{2}{*}{ Índice Fítness } & Control & $53,85 \pm 41,79$ & $56,92 \pm 28,27$ & 0,43 & 0,08 & 0,24 \\
\hline & Experimental & $59 \pm 31,11$ & $73,75 \pm 18,26$ & & & \\
\hline \multirow[t]{2}{*}{ Pulso UKK } & Control & $128,23 \pm 14,14$ & $128,15 \pm 17,63$ & 0,45 & $0,01 *$ & $0,01 *$ \\
\hline & Experimental & $129,87 \pm 18,84$ & $115,50 \pm 18,57$ & & & \\
\hline \multirow{2}{*}{$\begin{array}{l}\text { Pulso UKK } \\
\text { Recuperación }\end{array}$} & Control & $118,01 \pm 15,44$ & $116,92 \pm 22,19$ & $0,04 *$ & 0,31 & 0,49 \\
\hline & Experimental & $104 \pm 15,21$ & $97,88 \pm 19,22$ & & & \\
\hline
\end{tabular}

$* \mathrm{p}<0,05 ; \mathrm{VO}_{2 \max }$ : Consumo de Oxígeno estimada de forma indirecta; Pulso UKK: Pulsaciones cardíacas al terminar la prueba; Pulso UKK Recuperación: Pulsaciones cardíacas tras 1'de recuperación.

\section{Resistencia Muscular}

Tampoco ha habido mejoras significativas para la RM ni para la prueba de mancuerna (flexión-extensión del brazo con mancuerna) ni para la prueba de sentadilla (test de sentarselevantarse de la silla) (tabla 4).

Tabla 4. Efecto de un programa de ejercicio físico supervisado y no supervisado de cinco semanas de duración sobre las pruebas de Resistencia Muscular.

\begin{tabular}{|c|c|c|c|c|c|c|}
\hline Pruebas & Grupo & Pre & Post & $\begin{array}{c}P \text { para efecto } \\
\text { Grupo }\end{array}$ & $\begin{array}{c}P \text { para efecto } \\
\text { Tiempo }\end{array}$ & $\begin{array}{c}P \text { para efecto } \\
\text { interacción Grupo } \\
\text { x Tiempo }\end{array}$ \\
\hline \multirow[t]{2}{*}{ Mancuerna } & Control & $12,31 \pm 3,19$ & $13,15 \pm 4,77$ & $0,03 *$ & $0.01 *$ & 0,10 \\
\hline & Experimental & $14,50 \pm 3,38$ & $17,88 \pm 2,80$ & & & \\
\hline \multirow[t]{2}{*}{ Sentadilla } & Control & $14,85 \pm 3,28$ & $15,38 \pm 3,38$ & 0,11 & 0,39 & 0,83 \\
\hline & Experimental & $17,38 \pm 3,99$ & $18,25 \pm 5,89$ & & & \\
\hline
\end{tabular}

$* \mathrm{p}<\overline{<0,05 . \text { Los datos de Mancuerna y de Sentadilla del Pre y del Post corresponden al número de repeticiones }}$ realizados en estos tests.

\section{Composición Corporal}

No se han encontrado diferencias significativas para la CC en las variables Peso, IMC y porcentaje de grasa corporal (Tabla 5).

Tabla 5. Efecto de un programa de ejercicio físico supervisado y no supervisado de cinco semanas de duración sobre la Composición Corporal.

\begin{tabular}{|c|c|c|c|c|c|c|}
\hline Prueba & Grupo & Pre & Post & $\begin{array}{c}P \text { para efecto } \\
\text { Grupo }\end{array}$ & $\begin{array}{c}P \text { para efecto } \\
\text { Tiempo }\end{array}$ & $\begin{array}{c}\text { P para efecto } \\
\text { interacción Grupo } \\
\times \text { Tiempo }\end{array}$ \\
\hline \multirow[t]{2}{*}{ Peso (kg) } & Control & $68,49 \pm 7,32$ & $67,90 \pm 7,10$ & $0,05 *$ & 0,08 & 0.94 \\
\hline & Experimental & $60,56 \pm 10,52$ & $60,01 \pm 10,30$ & & & \\
\hline \multirow[t]{2}{*}{ IMC } & Control & $26,63 \pm 3,39$ & $26,43 \pm 3,42$ & 0,38 & $0,03 *$ & 0,69 \\
\hline & Experimental & $25,20 \pm 4,18$ & $24,91 \pm 4,19$ & & & \\
\hline \multirow[t]{2}{*}{ \% Grasa } & Control & $34,45 \pm 5,13$ & $34,22 \pm 5,79$ & 0,40 & 0,99 & 0,43 \\
\hline & Experimental & $31,81 \pm 7,42$ & $32,05 \pm 7,50$ & & & \\
\hline
\end{tabular}

* p < 0,05; IMC: Índice de Masa Corporal; \% Grasa: Porcentaje de Grasa Corporal. 
Santos-Olmo, P. A.; Jiménez-Díaz, J. F., y Rioja-Collado, N. (2019). Efecto de un programa de ejercicio de corta duración sobre la condición física y la calidad de vida en mujeres supervivientes de cáncer de mama del ámbito rural: Estudio Piloto. RICYDE. Revista internacional de ciencias del deporte. 56(15), 171-186.

https://doi.org/10.5232/ricyde2019.05604

\section{Calidad de Vida}

Se analizaron las escalas funcionales y los síntomas del cuestionario EORTC QLQ-C30. Para las escalas funcionales y la escala global de salud física, los resultados (Tabla 6) indican que, si bien el grupo experimental obtiene mejores puntuaciones, también lo hace el grupo control, no resultando significativas para $\mathrm{p}<0,05$ en ninguna de ellas.

En relación a los síntomas, menores puntuaciones indican menor limitación o impacto del síntoma. Aunque síntomas como fatiga, dolor pérdida del apetito mejoran para el grupo experimental, no se encuentran mejoras significativas para ninguna de las variables para $\mathrm{p}<$ 0,05 (Tabla 7).

Tabla 6. Efecto de un programa de ejercicio físico supervisado y no supervisado de cinco semanas de duración sobre la escala global de salud física y las escalas funcionales del cuestionario EORTC QLQ-C30.

\begin{tabular}{|c|c|c|c|c|c|c|}
\hline Escalas & Grupo & Pre & Post & $\begin{array}{c}P \text { para efecto } \\
\text { Grupo }\end{array}$ & $\begin{array}{c}P \text { para efecto } \\
\text { Tiempo }\end{array}$ & $\begin{array}{c}P \text { para efecto } \\
\text { interacción Grupo } \\
\text { x Tiempo }\end{array}$ \\
\hline \multirow{2}{*}{$\begin{array}{l}\text { Estado } \\
\text { General Salud }\end{array}$} & Control & $67,94 \pm 14,76$ & $67,46 \pm 14,88$ & \multirow[t]{2}{*}{0,66} & \multirow[t]{2}{*}{$0,00 *$} & \multirow[t]{2}{*}{0,16} \\
\hline & Experimental & $66,66 \pm 16,06$ & $79,17 \pm 14,08$ & & & \\
\hline \multirow[t]{2}{*}{ Aspecto Físico } & Control & $87,18 \pm 8,37$ & $91,79 \pm 7,28$ & \multirow[t]{2}{*}{0,34} & \multirow[t]{2}{*}{$0,00 *$} & \multirow[t]{2}{*}{0,39} \\
\hline & Experimental & $90,83 \pm 3,45$ & $93,33 \pm 3,56$ & & & \\
\hline \multirow[t]{2}{*}{ Autonomía } & Control & $89,74 \pm 17,39$ & $91,03 \pm 14,61$ & \multirow[t]{2}{*}{0,42} & \multirow[t]{2}{*}{0,66} & \multirow[t]{2}{*}{0,91} \\
\hline & Experimental & $93,75 \pm 12,40$ & $95,83 \pm 11,78$ & & & \\
\hline \multirow[t]{2}{*}{ Emocional } & Control & $77,56 \pm 23,41$ & $86,54 \pm 16,50$ & \multirow[t]{2}{*}{0,62} & \multirow[t]{2}{*}{0,11} & \multirow[t]{2}{*}{0,43} \\
\hline & Experimental & $84,37 \pm 16,32$ & $87,50 \pm 17,81$ & & & \\
\hline \multirow[t]{2}{*}{ Cognitiva } & Control & $71,79 \pm 21,92$ & $75,64 \pm 27,73$ & \multirow[t]{2}{*}{0,43} & \multirow[t]{2}{*}{0,65} & \multirow[t]{2}{*}{0,65} \\
\hline & Experimental & $81,25 \pm 13,90$ & $81,25 \pm 22,60$ & & & \\
\hline \multirow[t]{2}{*}{ Social } & Control & $73,08 \pm 22,08$ & $84,62 \pm 18,58$ & \multirow[t]{2}{*}{$0,05 *$} & \multirow[t]{2}{*}{$0,01 *$} & \multirow[t]{2}{*}{0,23} \\
\hline & Experimental & $91,67 \pm 12,59$ & $95,83 \pm 11,78$ & & & \\
\hline
\end{tabular}

$* \mathrm{p}<0,05$

Tabla 7. Efecto de un programa de ejercicio físico supervisado y no supervisado de cinco semanas de duración sobre las escalas de síntomas del cuestionario EORTC QLQ-C30.

\begin{tabular}{|c|c|c|c|c|c|c|}
\hline Síntomas & Grupo & Pre & Post & $\begin{array}{c}P \text { para efecto } \\
\text { Grupo }\end{array}$ & $\begin{array}{c}P \text { para efecto } \\
\text { Tiempo }\end{array}$ & $\begin{array}{c}P \text { para efecto } \\
\text { interacción Grupo } \\
\text { x Tiempo }\end{array}$ \\
\hline \multirow[t]{2}{*}{ Fatiga } & Control & $26,50 \pm 16,06$ & $27,35 \pm 19,03$ & 0,14 & 0,51 & 0,38 \\
\hline & Experimental & $19,44 \pm 16,53$ & $13,89 \pm 14,24$ & & & \\
\hline \multirow[t]{2}{*}{ Náusea } & Control & $10,26 \pm 27,67$ & $1,28 \pm 4,62$ & 0,26 & 0,37 & 0,37 \\
\hline & Experimental & $0,0 \pm 0,0$ & $0,0 \pm 0,0$ & & & \\
\hline \multirow[t]{2}{*}{ Dolor } & Control & $19,23 \pm 13,34$ & $19,23 \pm 14,97$ & 0,54 & 0,44 & 0,44 \\
\hline & Experimental & $20,83 \pm 17,77$ & $10,42 \pm 12,40$ & & & \\
\hline \multirow[t]{2}{*}{ Disnea } & Control & $17,95 \pm 17,29$ & $12,82 \pm 16,87$ & 0,15 & 0,89 & 0,22 \\
\hline & Experimental & $4,17 \pm 11,78$ & $8,33 \pm 15,43$ & & & \\
\hline \multirow{2}{*}{ Insomnio } & Control & $38,46 \pm 35,60$ & $33,33 \pm 33,33$ & 0,28 & $0,05 *$ & 0,29 \\
\hline & Experimental & $29,17 \pm 37,53$ & $12,50 \pm 17,25$ & & & \\
\hline \multirow[t]{2}{*}{ Pérdida apetito } & Control & $2,56 \pm 9,24$ & $2,56 \pm 9,24$ & 0,37 & 0,48 & 0,48 \\
\hline & Experimental & $8,33 \pm 15,43$ & $4,17 \pm 11,78$ & & & \\
\hline \multirow[t]{2}{*}{ Estreñimiento } & Control & $28,21 \pm 38,11$ & $25,64 \pm 38,85$ & 0,31 & 0,83 & 0,83 \\
\hline & Experimental & $12,50 \pm 24,80$ & $12,50 \pm 24,80$ & & & \\
\hline \multirow[t]{2}{*}{ Diarrea } & Control & $7,69 \pm 19,97$ & $2,56 \pm 9,24$ & 0,26 & 0,44 & 0,44 \\
\hline & Experimental & $0,0 \pm 0,0$ & $0,0 \pm 0,0$ & & & \\
\hline \multirow[t]{2}{*}{ Impacto Financiero } & Control & $17,95 \pm 25,87$ & $7,69 \pm 14,61$ & 0,58 & $0,04 *$ & 0,82 \\
\hline & Experimental & $12,50 \pm 24,80$ & $4,17 \pm 11,78$ & & & \\
\hline
\end{tabular}

$* \mathrm{p}<0,05$. 
Santos-Olmo, P. A.; Jiménez-Díaz, J. F., y Rioja-Collado, N. (2019). Efecto de un programa de ejercicio de corta duración sobre la condición física y la calidad de vida en mujeres supervivientes de cáncer de mama del ámbito rural: Estudio Piloto. RICYDE. Revista internacional de ciencias del deporte. 56(15), 171-186.

https://doi.org/10.5232/ricyde2019.05604

\section{Discusión}

El ejercicio físico se ha revelado como una herramienta clave en la recuperación y mantenimiento de las cualidades físicas y psicológicas saludables y por consiguiente, en la mejora de la CV de las SCM. Foley y Hasson (2016) mejoraron de forma significativa la movilidad, la CA, la RM del tren inferior y superior, la flexibilidad del tren superior y equilibrio en tan solo 12 semanas de un programa con dos sesiones supervisadas de 90 minutos. La muestra, que tenía una media de 59,7 años de edad, tenían en la prueba de seis minutos caminando, valores similares a la población por encima de 80 años. Tras la intervención, habían ganado 10 años, estando cercanos a la población de 70-80 años (Foley y Hasson, 2016).

Aun así, las SCM realizan poca o nula actividad física de ocio (Olson y col., 2014) no llegando en la mayor parte de casos a las recomendaciones de ejercicio físico saludable (Rogers y col., 2015).

El $47 \%$ de la muestra de este estudio eran mujeres jóvenes premenopaúsicas ( $<51$ años), que con frecuencia se interesan más por su CV e imagen corporal (Howard-Anderson, Ganz, Bower, y Stanton, 2012). Pese a que el ejercicio físico se muestra como una herramienta segura después del tratamiento (Benton y col., 2014), que puede mitigar el dolor continuo que sufren las SCM (Forsythe, 2013) y ser un elemento muy atractivo de participación, es destacable la baja tasa de reclutamiento en ciertas localidades, pudiendo explicarse por el déficit en conductas activas, el escaso apoyo de la familia-entorno hacia la práctica de ejercicio físico (Vallance, Lavallee, Culos-Reed, \& Trudeau, 2012), así como la obligación de tener que desplazarse hasta la instalación deportiva.

Con la intención de cambiar estos comportamientos y mejorar la motivación y adherencia en estas zonas rurales, se optó por un programa que fuera de corta duración, de tan solo cinco semanas y con una estructura del programa en sesiones supervisadas y no supervisadas. Si bien una intervención realizada in situ y supervisada ha demostrado mejores resultados (Buffart y col., 2017; Meneses-Echavez, González-Jiménez, Correa, y Ramírez-Vélez, 2014) que aquel que se realiza en el domicilio (Musanti, 2012), el ejercicio no supervisado puede obtener beneficios a largo plazo para SCM a nivel funcional y morfológico (IMC y pliegues cutáneos) (Mascherini y col., 2018).

Así, la práctica de ejercicio físico continuado y planificado, supervisado o no, conlleva beneficios físicos y morfológicos para las mujeres SCM, y en zonas rurales, donde es posible que carezcan de espacios deportivos equipados, las sesiones no supervisadas pueden ser una herramienta que facilite la participación en programas prolongados.

No se han constatado mejoras significativas para ninguna de las variables para la CA. A pesar de ello, es de gran relevancia clínica, el aumento del 26,17 \% del $\mathrm{VO}_{2 \max }$ del grupo experimental, favoreciendo el pronóstico de la enfermedad (Meyer, Welter, Scharhag, y Kindermann, 2003). Algo similar ha ocurrido con el Índice Fítness también para el grupo experimental que ha pasado del escalón más bajo, a "algo por debajo de la media" con 73,75 puntos (Oja y col., 2013).

Este programa de tan sólo cinco semanas, ha conseguido para el grupo experimental aumentar su $\mathrm{VO}_{2 \max }$ por encima de $20 \mathrm{ml}$. min. kg, y su Índice Fitness por encima de 70 puntos. Por debajo de $20 \mathrm{ml}$. min. kg para las mujeres y $30 \mathrm{ml}$. min. kg para los hombres, el riesgo de muerte de todas las causas y por enfermedad coronaria es mayor (Oja y col., 2013).

Estos datos están en consonancia con estudios que establecen que si bien para las SCM se pueden seguir las recomendaciones del Colegio Americano de Medicina del Deporte, de una frecuencia cardíaca máxima del 60 - 90 \% (Chamorro y Pérez, 2012), estas recomendaciones 
Santos-Olmo, P. A.; Jiménez-Díaz, J. F., y Rioja-Collado, N. (2019). Efecto de un programa de ejercicio de corta duración sobre la condición física y la calidad de vida en mujeres supervivientes de cáncer de mama del ámbito rural: Estudio Piloto. RICYDE. Revista internacional de ciencias del deporte. 56(15), 171-186.

https://doi.org/10.5232/ricyde2019.05604

no se ajustan a su perfil cardiorrespiratorio por debajo de lo saludable. Sería conveniente establecer pautas específicas, ajustándose más a la realidad que ofrecen datos como el $\mathrm{VO}_{2 \max }$, la frecuencia cardíaca de reserva, o la escala de esfuerzo percibido (Gil-Rey, Quevedo-Jerez, Maldonado-Martin, y Herrero-Román, 2015).

Hay suficiente evidencia científica para determinar que en SCM la capacidad aeróbica mejora de forma significativa después de la intervención (Battaglini y col., 2014; Schmitz y col., 2010), aunque en este estudio no se ha conseguido, pudiendo deberse posiblemente a la corta duración del tratamiento.

En el trabajo de RM, los ejercicios se idearon con especial atención a la zona del hombro y del brazo y la amplitud del movimiento se limitó en caso de dolor o molestia. La intensidad siempre fue moderada, en el rango de 10 - 14 de la Escala de Borg (Gil-Rey y col., 2015) , y la carga solo fue incrementada un $5-10 \%$ cuando se podía realizar todas las series y repeticiones de una sesión con soltura (Benton y col., 2014), sin provocar molestia durante la sesión, ni en el día posterior al entrenamiento.

Después de cinco semanas de intervención, tampoco se han obtenido mejoras significativas para las dos variables de estudio de la RM, la prueba de mancuernas (test funcional de flexión de brazos), y la prueba de sentadillas (Chair stand test).

A pesar de no encontrarse diferencias significativas, es interesante la mejora del $18.91 \%$ del grupo experimental para la prueba de mancuernas, ya que las SCM a menudo, padecen de una limitación funcional en los miembros superiores, sobre todo, en la movilidad y fuerza del hombro afectado, conformando el mayor grado discapacitante (Harrington, Padua, Battaglini, y Michener, 2013). Los trabajos de Nauman y col. (2012), con un programa similar al nuestro, pero de ocho semanas de duración consiguieron mejorar de forma significativa $(\mathrm{p}<0.05)$, la fuerza para el tren superior e inferior y el $\mathrm{VO}_{2 \max }$ (Naumann y col., 2012).

A este respecto, Hagstrom; Shorter, y Marshall (2017), confirmaron las diferencias significativas en SCM de los niveles de fuerza en ambos brazos, además de que esas diferencias no pudieron ser corregidas tras una intervención de 16 semanas de trabajo de resistencia muscular bilateral (Hagstrom, Shorter, y Marshall, 2017).

La sarcopenia es una peculiaridad prominente de la caquexia que padecen los pacientes de cáncer, que puede ser la consecuencia de una disfuncionalidad mitocondrial, disminución de la capacidad oxidativa y de fuerza muscular (Khamoui y Kim, 2012), entrando en un ciclo progresivo de menor músculo esquelético, menor capacidad funcional y mayor fatiga. El ejercicio a través de un trabajo multivariante aeróbico y de resistencia muscular, posee un potente efecto antiinflamatorio y antioxidante, atenúa el desgaste y degradación muscular y mejora la síntesis de proteínas a través del aumento inducido de los niveles de IGF-1 y reduce la señalización de miostatina (Bowen, Schuler, y Adams, 2015).

Este programa no ha conseguido mejoras significativas para la CC. Estudios con una estructura similar de trabajo de CA y RM para SCM $(\mathrm{p}=0,90)$ después de 12 semanas (Musanti, 2012), o con una muestra compuesta de SCM y población sana (Simonavice y col., 2011), no obtuvieron beneficios sobre la CC. Estos datos son discordantes con estudios como los de Fernández Lao y col. (2013) en los que con ocho semanas de un programa de actividad multilateral consiguió una mejora significativa en el porcentaje de grasa $(p=0,01)$ (Fernández-Lao y col., 2013). Es posible que la bioimpedancia no sea un instrumento capaz de medir los pequeños cambios de grasa acontecidos. Según los trabajos de Sillanpää y col., (2014), la densitometría es más sensible para aportar datos favorables de una muestra sometida a un programa de corta duración (Sillanpää y col., 2014). 
Santos-Olmo, P. A.; Jiménez-Díaz, J. F., y Rioja-Collado, N. (2019). Efecto de un programa de ejercicio de corta duración sobre la condición física y la calidad de vida en mujeres supervivientes de cáncer de mama del ámbito rural: Estudio Piloto. RICYDE. Revista internacional de ciencias del deporte. 56(15), 171-186.

https://doi.org/10.5232/ricyde2019.05604

Según Ruiz Casado y col. (2014), un alto porcentaje de supervivientes de cáncer son obesos (IMC $>30 \mathrm{~kg} / \mathrm{m}^{2}$ ) (Ruiz-Casado y col., 2014). La muestra del estudio tenía un perfil de IMC y porcentaje de grasa por encima del 25 para el IMC y del $30 \%$ para el porcentaje de grasa, traduciéndose en un marcado perfil de sobrepeso. Se hace necesario implantar programas de ejercicio físico junto con el seguimiento de una dieta equilibrada.

No se han encontrado mejoras significativas para para la CV medido a través del cuestionario EORTC QLQ-C30. A pesar de ello, las puntuaciones de los síntomas de la fatiga o del dolor del grupo experimental han sido muy positivas con una mejora del 13,12 \% y del 49,98\% respectivamente. Se tratan de síntomas fundamentales del cáncer y pueden explicar en parte, la variabilidad de ánimo deprimido en los supervivientes de cáncer (Galiano-Castillo y col., 2014). Sería interesante que se observaran estos síntomas en un tratamiento de mayor duración y muestra. La baja muestra y la falta de cegamiento de la intervención, determina para algunos autores, el efecto del ejercicio sobre la CV posea una evidencia baja-moderada para mujeres con cáncer durante la terapia (Furmaniak, Menig, y Markes, 2016). Aunque resulta complicado cegar el grupo de ejercicio ya que es conocido por parte de la participante. En un estudio con un programa que combinaba la CA y RM (Saarto y col., 2012), tampoco se encontraron diferencias en cuanto a las escalas medidas con este cuestionario, a pesar de consistir en un programa de un año de duración.

En este estudio, ambos grupos en las evaluaciones iniciales presentaban puntuaciones por encima de los valores de referencia del cuestionario. La alta puntuación inicial y una muestra pequeña pueden haber ocasionado que las desviaciones típicas sean altas, y por tanto, la incertidumbre a la hora de analizar los datos sea mayor. Esta puntuación de la evaluación inicial, se encuentra en consonancia con un estudio (Bantema-Joppe y col., 2015) con SCM de hasta cinco años después de la cirugía conservadora y tratamiento de radioterapia. Las mayores mejoras del cuestionario EORTC QLQ-C30 se conseguían en el primer año tras el tratamiento, y hacia los tres años, las puntuaciones eran superiores a los de referencia (alrededor de 90 puntos) y equiparables a la población en general. Los datos aportados por el cuestionario EORTC QLC-C30 versión 3.0, podrían ser complementados por el cuestionario FACT 4 (Functional Assessment of Cancer Treatment), instrumento sencillo y de fácil comprensión que es utilizado en pacientes oncológicos.

Con un programa de sesiones supervisadas y no supervisadas de cinco semanas de duración, solo se observa una tendencia positiva pero no una mejora. Es probable que una mayor muestra y duración hubiera podido mejorar de forma significativa la condición física y calidad de vida de las supervivientes del cáncer y los efectos del tratamiento, como así lo indica un reciente metaanálisis (Fuller, Hartland, Maloney, y Davison, 2018).

\section{Conclusión}

Un programa de ejercicio supervisado y no supervisado de cinco semanas de duración, siguiendo las recomendaciones de la Organización Mundial de la Salud de al menos, 150 minutos semanales de actividad física moderada, ha sido un estímulo bajo e insuficiente para conseguir constatar efectos en la mejora de la condición física y calidad de vida en supervivientes de cáncer de mama del ámbito rural, aunque se aprecian cambios positivos de un $26,17 \%$ de mejora en el $\mathrm{VO}_{2 \max }$ y un $18,91 \%$ de la fuerza en brazos en el grupo que realizó el programa de entrenamiento. 


\section{Referencias}

Aaronson, N. K.; Ahmedzai, S.; Bergman, B.; Bullinger, M.; Cull, A.; Duez, N. J.; Filiberti, A.; Flechtner, H.; Fleishman, S. B.; de Haes, J.C.; Kaasa, S.; Klee, M.; Osoba, D; Razavi, D.; Rofe, P. B.; Schraub, S.; Sneeuw, K.; Sullivan, M., \& Takeda, F. (1993). The European Organization for Research and Treatment of Cancer QLQ-C30: a quality-of-life instrument for use in international clinical trials in oncology. Journal of the National Cancer Institute, 85(5), 365-376.

Bantema-Joppe, E. J.; de Bock, G. H.; Woltman-van Iersel, M.; Busz, D. M.; Ranchor, A. V.; Langendijk, J. A.; Maduro, J. H., \& van den Heuvel, E. R. (2015). The impact of age on changes in quality of life among breast cancer survivors treated with breastconserving surgery and radiotherapy. British Journal of Cancer, 112(4), 636-643. https://doi.org/10.1038/bjc.2014.632

Battaglini, C. L.; Mills, R. C.; Phillips, B. L.; Lee J. T.; Story, C. E.; Nascimento, M. G., \& Hackney, A. C. (2014). Twenty-five years of research on the effects of exercise training in breast cancer survivors: A systematic review of the literature. World Journal of Clinical Oncology, 5(2), 177-190.

https://doi.org/10.5306/wjco.v5.i2.177

Befort, C.; Bennett, L.; Christifano, D.; Klemp, J. R., \& Krebill, H. (2014). Effective recruitment of rural breast cancer survivors into a lifestyle intervention. Psychooncology. 24(4), 487-490.

https://doi.org/10.1002/pon.3614

Benton, M.; Schlairet, M., \& Gibson, D. (2014). Change in quality of life among breast cancer survivors after resistance training: is there an effect of age? Journal of Aging and Physical Activity, 22(2), 178-185.

https://doi.org/10.1123/japa.2012-0227

Bowen, T. S.; Schuler, G., \& Adams, V. (2015). Skeletal muscle wasting in cachexia and sarcopenia: molecular pathophysiology and impact of exercise training. Journal of Cachexia, Sarcopenia and Muscle, 6(3), 197-207.

https://doi.org/10.1002/jcsm.12043

Buffart, L. M.; Kalter, J.; Sweegers, M. G.; Courneya, K. S.; Newton, R. U.; Aaronson, N., et al. (2016). Effects and moderators os exercise on quality of life and psysical function in patients with cancer: An individual patient data meta-analysis of 34 RCTs. Cancer Treatment Reviews, 52, 91-104.

https://doi.org/10.1016/j.ctrv.2016.11.010

Bydoun, M.; Marcato, P., \& Dellaire, G. (2013). Breast Cancer Genomics. En G. B. J. N. A. R. J. Dellaire (Ed.), Cancer Genomics from bench to personalized medicine ( $1^{\text {a }}$ ed., pp. 213-232). San Diego: Elsevier.

Campbell, K. L.; Pusic, A. L., Zucker, D. S.; McNeely, M. L.; Binkley, J. M.; Cheville, A. L., \& Harwood, K. J. (2012). A prospective model of care for breast cancer rehabilitation: Function. Cancer, 118(8 Suppl), 2300-2311. https://doi.org/10.1002/cncr.27464

Chamorro, C., y Pérez, M. (2012). Prescripción del ejercicio físico para enfermos de cáncer. En S. G. N. Márquez (Ed.), Actividad física y salud (pp. 391-401).

Cramp, F., \& Byron-Daniel, J. (2012). Exercise for the management of cancer-related fatigue in adults. The Cochrane database of systematic reviews, 11. https://doi.org/10.1002/14651858.cd006145

Dulko, D.; Pace, C. M.; Dittus, K. L.; Sprague, B. L.; Pollack, L. A.; Hawkins, N. A., \& Geller, B. M. (2013). Barriers and facilitators to implementing cancer survivorship care plans. Oncology Nursing Forum, 40(6), 575-580.

https://doi.org/10.1188/13.ONF.575-580 
Santos-Olmo, P. A.; Jiménez-Díaz, J. F., y Rioja-Collado, N. (2019). Efecto de un programa de ejercicio de corta duración sobre la condición física y la calidad de vida en mujeres supervivientes de cáncer de mama del ámbito rural: Estudio Piloto. RICYDE. Revista internacional de ciencias del deporte. 56(15), 171-186.

https://doi.org/10.5232/ricyde2019.05604

Fernández-Lao, C.; Cantarero-Villanueva, I.; Ariza-García, A.; Courtney, C.; Fernández de las Peñas, C., y Arroyo-Morales, M. (2013). Water versus land-based multimodal exercise program effects on body composition in breast cancer survivors: a controlled clinical trial. Supportive Care in Cancer, 21(2), 521-530.

https://doi.org/10.1007/s00520-012-1549-x

Foley, M. P., \& Hasson, S. M. (2016). Effects of a Community-Based Multimodal Exercise Program on Health-Related Physical Fitness and Physical Function in Breast Cancer Survivors: A Pilot Study. Integrative Cancer Therapies, 15(4), 446-454. https://doi.org/10.1177/1534735416639716

Forsythe, L. P.; Alfano, C. M.; George, S. M.; McTiernan, A.; Baumgartner, K. B.; Bernstein, L., \& Ballard-Barbash, R. (2013). Pain in long-term breast cancer survivors: the role of body mass index, physical activity, and sedentary behavior. Breast Cancer Research and Treatment, 137(2), 617-630. https://doi.org/10.1007/s10549-012-2335-7

Fuller, J. T.; Hartland, M. C.; Maloney, L. T., \& Davison, K. (2018). Therapeutic effects of aerobic and resistance exercises for cancer survivors: a systematic review of metaanalyses of clinical trials. British Journal of Sports Medicine. Published Online First: 16 March 2018.

https://doi.org/10.1136/bjsports-2017-098285

Furmaniak, A. C.; Menig, M., \& Markes, M. H. (2016). Exercise for women receiving adjuvant therapy for breast cancer. Cochrane Database of Systematic Review, 9, CD005001.

https://doi.org/10.1002/14651858.CD005001.pub3

Galiano-Castillo, N.; Ariza-García, A.; Cantarero-Villanueva, I.; Fernández-Lao, C.; DíazRodríguez, L., y Arroyo-Morales, M. (2014). Depressed mood in breast cancer survivors: associations with physical activity, cancer-related fatigue, quality of life, and fitness level. European Journal of Oncology Nursing, 18, 206-210.

https://doi.org/10.1016/j.ejon.2013.10.008

Gewefel, H., \& Salhia, H. (2014). Breast cancer in adolescent and young adult women. Clinical Breast Cancer, 14(6), 390-395.

Gil-Rey, E.; Quevedo-Jerez, K.; Maldonado-Martin, S., \& Herrero-Román, F. (2015). Exercise intensity guidelines for cancer survivors: a comparison with reference values. International Journal of Sports Medicine. [Epub ahead of print].

https://doi.org/10.1055/s-0034-1389972

Hagstrom, A. D.; Shorter, K. A., \& Marshall, P. W. (2017). Changes in unilateral upper limb muscular strength and EMG activity following a 16 week strength training intervention survivors of breast cancer. Journal of Strength and Conditioning Research. [Epub ahead of print].

https://doi.org/10.1519/JSC.0000000000001890

Harrington, S.; Padua, D.; Battaglini, C., \& Michener, L. A. (2013). Upper extremity strength and range of motion and their relationship to function in breast cancer survivors. Physiothery Theory and Practice, 29(7), 513-520.

https://doi.org/10.3109/09593985.2012.757683

Herrero, F., y Pérez, M. (2011). Actividad física y cáncer. En J.A. Casajús y G. VicenteRodríguez (Eds.), Ejercico físico y salud en poblaciones especiales (Vol. 58, pp. 387410). Exernet Madrid: Colección ICD.

Howard-Anderson, J.; Ganz, P. A.; Bower, J. E., \& Stanton, A. L. (2012). Quality of life, fertility concerns, and behavioral health outcomes in younger breast cancer survivors: a systematic review. Journal of the National Cancer Institute, 104(5), 386-405. https://doi.org/10.1093/jnci/djr541 
Santos-Olmo, P. A.; Jiménez-Díaz, J. F., y Rioja-Collado, N. (2019). Efecto de un programa de ejercicio de corta duración sobre la condición física y la calidad de vida en mujeres supervivientes de cáncer de mama del ámbito rural: Estudio Piloto. RICYDE. Revista internacional de ciencias del deporte. 56(15), 171-186.

https://doi.org/10.5232/ricyde2019.05604

Khamoui, A. V., \& Kim, J. S. (2012). Candidate mechanisms underlying effects of contractile activity on muscle morphology and energetics in cancer cachexia. European Jornal of Cancer Care (Engl), 21(2), 143-157.

https://doi.org/10.1111/j.1365-2354.2011.01287.x

Ligibell, J. (2012). Lifestyle factors in cancer survivorship. Journal of Clinical Oncology, 30(30), 3697-3704.

https://doi.org/10.1200/jco.2012.42.0638

Lira, F. S.; Neto, J. C., \& Seelaender, M. (2014). Exercise training as treatment in cancer cachexia. Applied Physiology, Nutrition and Metabolism, 39(6), 679-686.

https://doi.org/10.1139/apnm-2013-0554

Mascherini, G.; Tosi, B.; Giannelli, C.; Grifoni, E.; Degl'innocenti, S., \& Galanti, G. (2018). Breast cancer: effectiveness of a one-year unsupervised exercise program. Journal of Sports Medicine and Physical Fitness. https://doi.org/10.23736/S0022-4707.18.08131-8

Meneses-Echavez, J.; González-Jiménez, E.; Correa, J., y Ramírez-Vélez, R. (2014). Intervenciones con actividad física supervisada en el manejo de la fatiga relacionada con el cáncer: una revisión sistemática. Nutrición Hospitalaria, 30(3), 486-497. https://doi.org/10.3305/nh.2014.30.3.7635

Meyer, T.; Welter, J. P.; Scharhag, J., \& Kindermann, W. (2003). Maximal oxygen uptake during field running does not exceed that measured during treadmill exercise. European Jorunal of Applied Physiology, 88(4-5), 387-389.

https://doi.org/10.1007/s00421-002-0718-x.

Minton, O.; Berger, A.; Barsevick, A.; Cramp, F.; Goedendorp, M.; Mitchell, S. A.; Stone, P. C. (2013). Cancer-related fatigue and its impact on functioning. Cancer, 119(Suppl 11), 2124-2130.

https://doi.org/10.1002/cncr.28058

Mirandola, D.; Miccinesi, G.; Muraca, M., Sgambati, E.; Monaci, M., \& Marini, M. (2014). Evidence for adapted physical activity as an effective intervention for upper limb mobility and quality of life inbreast cancer survivors. Journal of physical activity \& health, 11(4), 814-822.

https://doi.org/10.1123/jpah.2012-0119

Musanti, R. (2012). A study of exercise modality and physical self-esteem in breast cancer survivors. Medicine and Science in Sports and Exercise, 44(2), 352-361. https://doi.org/10.1249/MSS.0b013e31822cb5f2

Naumann, F.; Martin, E.; Philpott, M.; Smith, C.; Groff, D., \& Battaglini, C. (2012). Can counseling add value to an exercise intervention for improving quality of life in breast cancer survivors? A feasibility study. The Journal of Supportive Oncology, 10(5), 188194. https://doi.org/10.1016/j.suponc.2011.09.004

Oja, P.; Mänttäri, A.; M., Pokki, T.; Kukkonen-Harjula, K.; Laukkanen, R.; Malmberg, J. ; Miilunpalo, S., \& Suni, J. (2013). UKK Walk Test. Tester's guide (K. Ojala Ed. 4th revised edition). Tampere: UKK Institute for Health Promotion Research.

Olson, E.; Mullen, S.; Rogers, L.; Courneya, K.; Verhulst, S., \& McAuley, E. (2014). Meeting physical activity guidelines in rural breast cancer survivors. American Journal of Health Behavior, 38(6), 890-899.

Pedrero-Chamizo, R.; Gómez-Cabello, A.; Delgado, S.; Rodríguez-Llarena, S.; RodríguezMarroyo, J. A., Cabanillas, E.; Meléndez, A.; Vicente-Rodríguez, G.; Aznar, S.; Villa, G.; Espino, L.; Gusi, N.; Casajús, J.A.; Ara, I., y González-Gross, M. (2012). Physical fitness levels among independent non-institutionalized Spanish elderly: the elderly EXERNET multi-center study. Archives of Gerontology and Geriatrics, 55(2), 406-416. https://doi.org/10.1016/j.archger.2012.02.004 
Santos-Olmo, P. A.; Jiménez-Díaz, J. F., y Rioja-Collado, N. (2019). Efecto de un programa de ejercicio de corta duración sobre la condición física y la calidad de vida en mujeres supervivientes de cáncer de mama del ámbito rural: Estudio Piloto. RICYDE. Revista internacional de ciencias del deporte. 56(15), 171-186.

https://doi.org/10.5232/ricyde2019.05604

Rikli, R., \& Jones, C. (2013). Senior Fitness Test Manual (Second Edition). Champaign IL: Human Kinetics.

Rogers, L. Q.; Courneya, K. S.; Anton, P. M.; Hopkins-Price, P.; Verhulst, S.; Vicari, S. K.; Robbs, R. S.; Mocharnuk, R., \& McAuley, E. (2015). Effects of the BEAT Cancer physical activity behavior change intervention on physical activity, aerobic fitness, and quality of life in breast cancer survivors: a multicenter randomized controlled trial. Breast Cancer Research and Treatment, 149(1), 109-119. https://doi.org/10.1007/s10549-014-3216-z

Royo, A. (2011). Calidad de vida en pacientes intervenidas de cáncer de mama. Universitat Autónoma de Barcelona, Barcelona.

Ruiz-Casado, A.; Verdugo, A. S.; Solano, M. J.; Aldazabal, I. P.; Fiuza-Luces, C.; Alejo, L. B.; Padilla del Hierro, J. R.; Palomo, I.; Aguado-Arroyo, O.; Garatachea, N.; Cebolla, H., y Lucia, A. (2014). Objectively assessed physical activity levels in Spanish cancer survivors. Oncology Nursing Forum, 41(1), E12-20.

https://doi.org/10.1188/14.ONF.E12-E20

Saarto, T.; Penttinen, H.; Sievänen, H.; Kellokumpu-Lehtinen, P.; Hakamies-Blomqvist, L.; Nikander, R.; Huovinen, R.; Luoto, R.; Kautiainen, H.; Järvenpää, S.; Idman, I.; Utriainen, M.; Vehmanen, L.; Jääskeläinen, A. S.; Elme, A.; Ruohola, J.; Palva, T.; Vertio, H.; Rautalahti, M.; Fogelholm, M.; Blomqvist, C., \& Luoma, M. L. (2012). Effectiveness of a 12-month exercise program on physical performance and quality of life of breast cancer survivors. Anticancer Research, 32(9), 3875-3884.

Schmitz, K.; Courneya, K.; Matthews, C.; Demark-Wahnefried, W.; Galvão, D.; Pinto, B. ; Irwin, M.; Wolin, K.; Segal, R.; Lucia, A.; Scheneider, C.; Von Gruenigen, V., \& Schwartz, A. (2010). American College of Sports Medicine roundtable on exercise guidelines for cancer survivors. Medicine and Science in Sports and Exercise, 42(7), 1409-1426.

https://doi.org/10.1249/MSS.0b013e3181e0c112

Schoormans, D.; Czene, K.; Hall, P., \& Brandberg, Y. (2015). The impact of co-morbidity on health-related quality of life in breast cancer survivors and controls. Acta Oncológica, 54(5): 727-34. https://doi.org/10.3109/0284186X.2014.998277

Segal, R.; Zwaal, C.; Green, E.; Tomasone, J. R.; Loblaw, A.; Petrella, T.; \& Group, E. f. P. w. C. G. D. (2017). Exercise for people with cancer: a clinical practice guideline. Curr Oncol, 24(1), 40-46. https://doi.org/10.3747/co.24.3376

Sillanpää, E.; Cheng, S.; Häkkinen, K.; Finni, T.; Walker, S.; Pesola, A.; Ahtiainen, J.; Stenroth, L.; Selänne, H., \& Sipilä, S. (2014). Body composition in 18-to 88-year-old adults--comparison of multifrequency bioimpedance and dual-energy X-ray absorptiometry. Obesity, 22(1), 101-109.

https://doi.org/10.1002/oby.20583

Simonavice, E.; Pei-Yang, L.; Ilich, J. Z.; Jeong-Su, K., \& Panton, L. B. (2011). Body composition, muscular strength, and physical function in breast cancer survivors. International Journal of Body Composition Research 9(2), 57-64.

Speck, R. M.; Gross, C. R.; Hormes, J. M.; Ahmed, R. L.; Lytle, L. A.; Hwang, W.-T., \& Schmitz, K. H. (2010). Changes in the Body Image and Relationship Scale following a one-year strength training trial for breast cancer survivors with or at risk for lymphedema. Breast Cancer Research And Treatment, 121(2), 421-430. https://doi.org/10.1007/s10549-009-0550-7 
Santos-Olmo, P. A.; Jiménez-Díaz, J. F., y Rioja-Collado, N. (2019). Efecto de un programa de ejercicio de corta duración sobre la condición física y la calidad de vida en mujeres supervivientes de cáncer de mama del ámbito rural: Estudio Piloto. RICYDE. Revista internacional de ciencias del deporte. 56(15), 171-186. https://doi.org/10.5232/ricyde2019.05604

Stout, N. L.; Baima, J.; Swisher, A. K.; Winters-Stone, K. M., \& Welsh, J. (2017). A Systematic Review of Exercise Systematic Reviews in the Cancer Literature (20052017). PM R, 9(9S2), S347-S384. https://doi.org/10.1016/j.pmrj.2017.07.074.

Vallance, J.; Lavallee, C.; Culos-Reed, N., \& Trudeau, M. (2012). Predictors of physical activity among rural and small town breast cancer survivors: an application of the theory of planned behaviour. Psychology, Health and Medicine, 17(6), 685-697. https://doi.org/10.1080/13548506.2012.659745 